\title{
A!
}

This is an electronic reprint of the original article.

This reprint may differ from the original in pagination and typographic detail.

Kajala, Jussi; Massel, Francesco; Törmä, Päivi

\section{Expansion dynamics of the Fulde-Ferrell-Larkin-Ovchinnakov state}

Published in:

Physical Review A

DOI:

10.1103/PhysRevA.84.041601

Published: 10/10/2011

Document Version

Publisher's PDF, also known as Version of record

Please cite the original version:

Kajala, J., Massel, F., \& Törmä, P. (2011). Expansion dynamics of the Fulde-Ferrell-Larkin-Ovchinnakov state.

Physical Review A, 84(4), 1-4. [041601]. https://doi.org/10.1103/PhysRevA.84.041601

This material is protected by copyright and other intellectual property rights, and duplication or sale of all or part of any of the repository collections is not permitted, except that material may be duplicated by you for your research use or educational purposes in electronic or print form. You must obtain permission for any other use. Electronic or print copies may not be offered, whether for sale or otherwise to anyone who is not an authorised user. 


\title{
Expansion dynamics of the Fulde-Ferrell-Larkin-Ovchinnikov state
}

\author{
J. Kajala, ${ }^{1}$ F. Massel, ${ }^{2}$ and P. Törmä ${ }^{1, *}$ \\ ${ }^{1}$ Department of Applied Physics, Aalto University School of Science, P.O. Box 15100, FI-00076 Aalto, Finland \\ ${ }^{2}$ Low Temperature Laboratory, Aalto University School of Science, P.O. Box 15100, FI-00076 Aalto, Finland
}

(Received 8 July 2011; published 10 October 2011)

\begin{abstract}
We consider a two-component Fermi gas in the presence of spin imbalance, modeling the system in terms of a one-dimensional attractive Hubbard Hamiltonian initially in the presence of a confining trap potential. With the aid of the time-evolving block decimation method, we investigate the dynamics of the initial state when the trap is switched off. We show that the dynamics of a gas initially in the Fulde-Ferrel-Larkin-Ovchinnikov (FFLO) state is decomposed into the independent expansion of two fluids, namely the paired and the unpaired particles. In particular, the expansion velocity of the unpaired cloud is shown to be directly related to the FFLO momentum. This provides an unambiguous signature of the FFLO state in a remarkably simple way.
\end{abstract}

DOI: $10.1103 /$ PhysRevA.84.041601

PACS number(s): 67.85.Lm, 71.10.Fd, 73.20.Mf

Ultracold gases have provided experimental verification for several fundamental concepts of quantum physics. The direct access to momentum distribution and correlations via timeof-flight expansion has played a key role in many landmark experiments. Mapping of momentum to position after time of flight revealed Bose-Einstein condensation [1]. Coherence manifesting after expansion gave evidence of the phase of the condensate [2], and of the superfluid-Mott insulator transition [3]. Inversion of the aspect ratio of an expanding Fermi gas revealed hydrodynamic behavior [4,5]. A major goal is to observe the elusive Fulde-Ferrel-Larkin-Ovchinnikov (FFLO) state, which is at the heart of understanding the interplay between superconductivity and magnetism. While experiments in solid-state systems [6-8] and ultracold gases [9] are consistent with the state, an unambiguous observation is lacking.

The FFLO phase is characterized by the formation of pairs with nonzero overall momentum $[10,11]$. This property manifests itself through the appearance of an oscillating (superconducting) order parameter $\Psi(r) \propto \Delta_{q} \exp [i q r]$, where $q$ is the so-called FFLO momentum $(\hbar=1)$ [12]. It is given by the mismatch between the Fermi momenta of $N_{\uparrow}$ spin-up and $N_{\downarrow}$ spin-down particles: $q=k_{F \uparrow}-k_{F \downarrow}$. In contrast, zero-momentum $(q=0)$ pairs give the conventional BCS superconductor physics. Despite the lack of genuine longrange order, the FFLO state has been theoretically predicted to be especially stable in one-dimensional (1D) systems [13-15], as well as in quasi-1D systems [16-18]. Inspired by the first experiments in imbalanced atomic Fermi gases [19,20], various methods for detecting the FFLO state in ultracold gases have been proposed (see [21] and references therein). In this Rapid Communication we show via exact simulations that time-of-flight expansion provides a smoking gun signature of the FFLO state in one dimension; see Fig. 1. Expansion and consequent imaging of densities is a widely used basic technique in experiments with ultracold gases.

The characteristic parameters can be chosen so that the lattice model employed is a good approximation for a continuum model of a spin-imbalanced gas in one dimension in the strong interaction limit $[16,22,23]$. With appropriate mapping of the

*paivi.torma@aalto.fi parameters, in the strong interaction limit, our predictions are thus relevant for experiments in 1D potentials as in [9].

We consider the 1D Fermi-Hubbard Hamiltonian in the presence of an overall confining potential

$$
\begin{aligned}
H= & U \sum_{i} \hat{n}_{i \uparrow} \hat{n}_{i \downarrow}-J \sum_{i \sigma=\uparrow, \downarrow} c_{i \sigma}^{\dagger} c_{i+1 \sigma} \\
& + \text { H.c. }+\sum_{i, \sigma=\uparrow, \downarrow} V_{i} \hat{n}_{i \sigma},
\end{aligned}
$$

where $c_{i \sigma}^{\dagger}\left(c_{i \sigma}\right)$ creates (annihilates) a spin $\sigma$ particle at the lattice site $i \in\{1, L\}, J$ is the hopping constant, $U$ is the interaction strength between the two species, and $V_{i}$ is the strength of the trapping potential. For a harmonic potential, $V_{i}=V_{h o}(i-C)^{2}$, where $C$ denotes the trap center, and for a box potential $V_{i}$ is specified below.

To obtain the ground state and time evolution of the system, we have employed the (essentially) exact time-evolving block decimation (TEBD) algorithm [24] with Schmidt number $\Gamma=150$ and simulation time step $\Delta t=0.02 \frac{1}{J}$. As results from TEBD, we obtain the single-particle densities $n_{i \uparrow}(t)=$ $\left\langle\Phi(t)\left|c_{i \uparrow}^{\dagger} c_{i \uparrow}\right| \Phi(t)\right\rangle, n_{i \downarrow}(t)=\left\langle\Phi(t)\left|c_{i \downarrow}^{\dagger} c_{i \downarrow}\right| \Phi(t)\right\rangle$, the doublon density $n_{\uparrow \downarrow}(t)=\left\langle\Phi(t)\left|c_{i \uparrow}^{\dagger} c_{i \downarrow}^{\dagger} c_{i \uparrow} c_{i \downarrow}\right| \Phi(t)\right\rangle$, and the groundstate pair correlation $C_{i j}$ and its momentum transform $n_{k}$, which are given by

$$
\begin{aligned}
C_{i j} & =\left\langle\Phi\left|c_{i \uparrow}^{\dagger} c_{i \downarrow}^{\dagger} c_{j \downarrow} c_{j \uparrow}\right| \Phi\right\rangle, \\
n_{k} & =\frac{1}{2 L} \sum_{i, j}^{L} e^{\imath(i-j) k} C_{i j},
\end{aligned}
$$

where $\langle\Phi \| \Phi\rangle$ describes the quantum-mechanical average over the state $\Phi, i$ and $j$ are lattice site indices, $l$ is the imaginary unit, and $L$ is the size of the lattice.

We have experimented within TEBD parameter ranges $N_{\uparrow}=0-40, \quad N_{\downarrow}=0-40, \quad P=\frac{N_{\uparrow}-N_{\downarrow}}{N_{\uparrow}+N_{\downarrow}}=0.024-1, \quad L=80-$ $320, V_{h o}=0.02 J-0.0001 J$, and $\Gamma=80-200$, where $P$ is the polarization and $V_{h o}$ is the harmonic trapping strength. Many simulations were performed also using a box potential. Single runs with the larger parameters have been done in order to check that the qualitative description of the dynamics stays the same when changing the parameters. Indeed, the important 


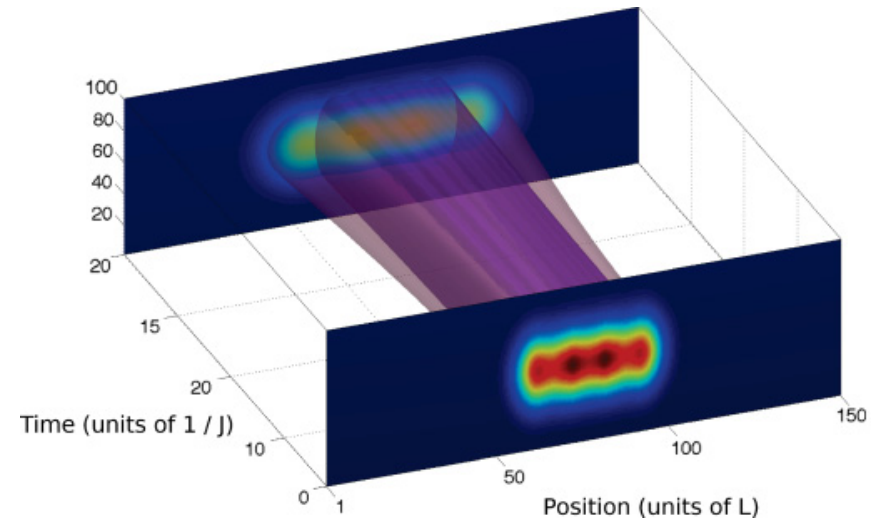

FIG. 1. (Color online) Expansion of a spin-density imbalanced Fermi gas in the 1D FFLO state after the confining potential has been switched off. Our exact simulations show an effective two-fluid behavior: pairs and unpaired particles expand with different velocities. Remarkably, the expansion velocity of the unpaired particles is directly related to the FFLO momentum and provides a straightforward way for observing the FFLO state.

characteristics of the dynamics are the same in all of the scenarios.

For simplicity we first consider here the box trap. In Fig. 2 we show results for 10 spin-up particles and 6 spin-down particles, with $U=-10 \mathrm{~J}$. The ground state is a $1 \mathrm{D}$ FFLO state,
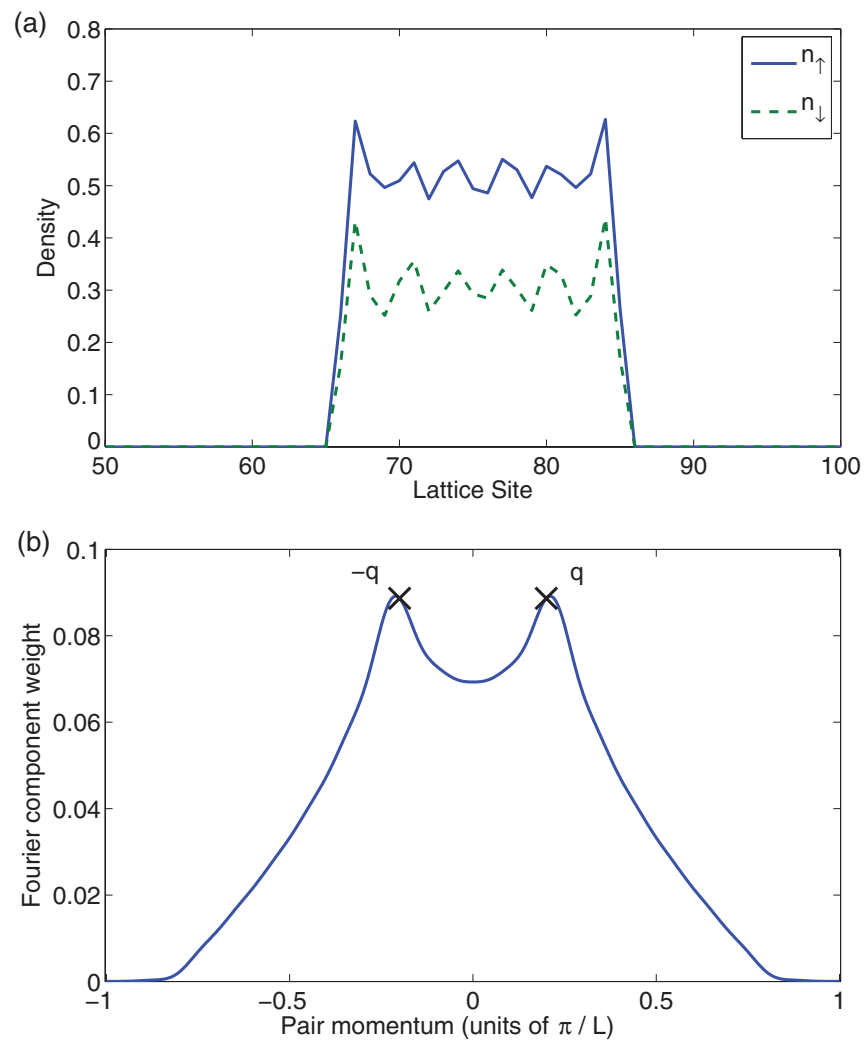

FIG. 2. (Color online) (a) The density profiles of up $\left(n_{\uparrow}\right)$ and down spins $\left(n_{\downarrow}\right)$ in the ground state when $N_{\uparrow}=10, N_{\downarrow}=6, U=$ $-10 \mathrm{~J}$, and there is to a good approximation an infinitely strong repulsive potential everywhere except at the lattice sites 66-85. (b) The pair momentum correlation function $n_{k}$ for the same state. There are peaks at the FFLO momenta $q= \pm\left(k_{F \uparrow}-k_{F \downarrow}\right)=0.2 \pi / L$.
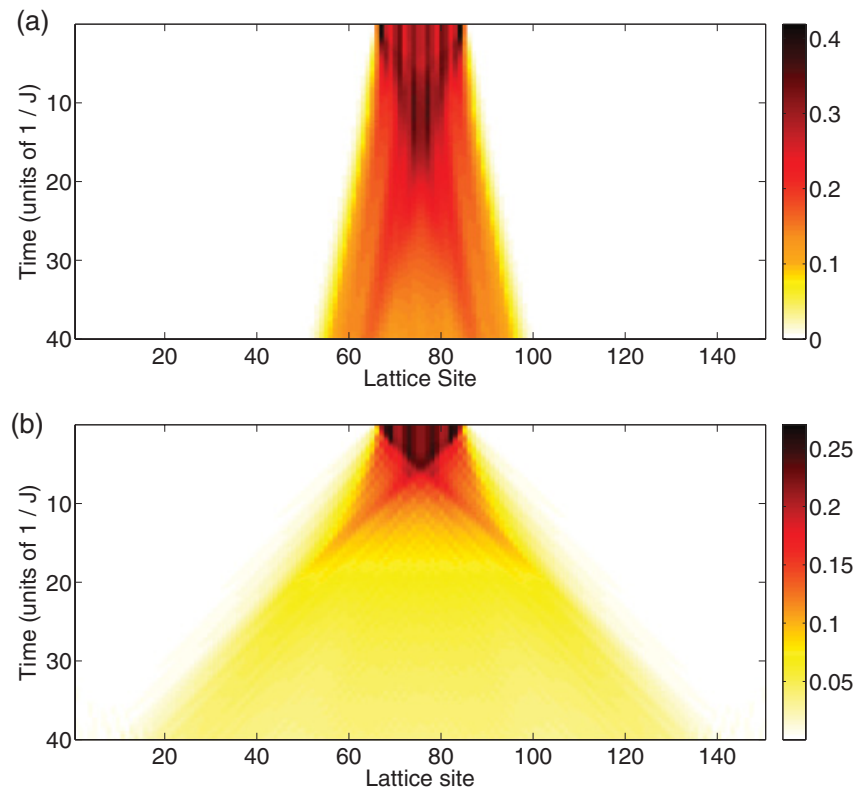

FIG. 3. (Color online) (a) The time development of the doublon density $n_{\uparrow \downarrow}$, corresponding to the ground state shown in Fig. 2. (b) The time development of the unpaired particle density $n_{\uparrow}-n_{\uparrow \downarrow}$.

characterized by a peak in the pair momentum distribution $n_{k}$ that coincides with the definition of the FFLO momentum, $q=k_{F \uparrow}-k_{F \downarrow}$. Figure 2(a) shows the ground-state density profile in which the small oscillations characterize the FFLO state. However, such delicate features are hard to resolve in ultracold-gas experiments, and therefore other signatures are needed. Figure 2(b) displays the pair momentum correlation function.

The dynamics after releasing the particles from the potential shows a striking two-fluid behavior. Pairs (doublons) and excess unpaired majority particles expand as effectively noninteracting fluids, as can be seen from Fig. 3. Indeed, we have compared the dynamics with strong interaction between the spins to the dynamics of noninteracting particles and verified that the doublons and unpaired particles in the strongly interacting limit expand qualitatively just like noninteracting particles would (see Figs. 2-5 in the supplementary material for the $U=0$ results [25]). The important difference is, however, that the velocity of the expansion is changed with respect to the noninteracting case. The doublons expand with velocities up to $\frac{4 J^{2}}{U} \sin \left(k_{F \downarrow}\right)$. And what is crucial for our proposal for observing the FFLO state is that the unpaired particles expand with velocities up to $2 J \sin (q)$, where $q$ is the FFLO momentum. In the $U \gg J$ case shown here, the unpaired particle velocity is larger than the paired one, but in general the relative velocities of paired versus unpaired particles is not essential for the method since the up and down components can be imaged separately (see, e.g., [9]).

A wave front corresponding to $q$ clearly separates from the rest of the unpaired particle cloud during the initial dynamics $\left(t=0-10 \frac{1}{J}\right)$, after which it moves with a constant velocity at the edge of the cloud; see Fig. 3(b). Therefore, by measuring the expansion velocity of the cloud edge $\left(v_{\text {exp }}\right)$, e.g., from the 
(a)

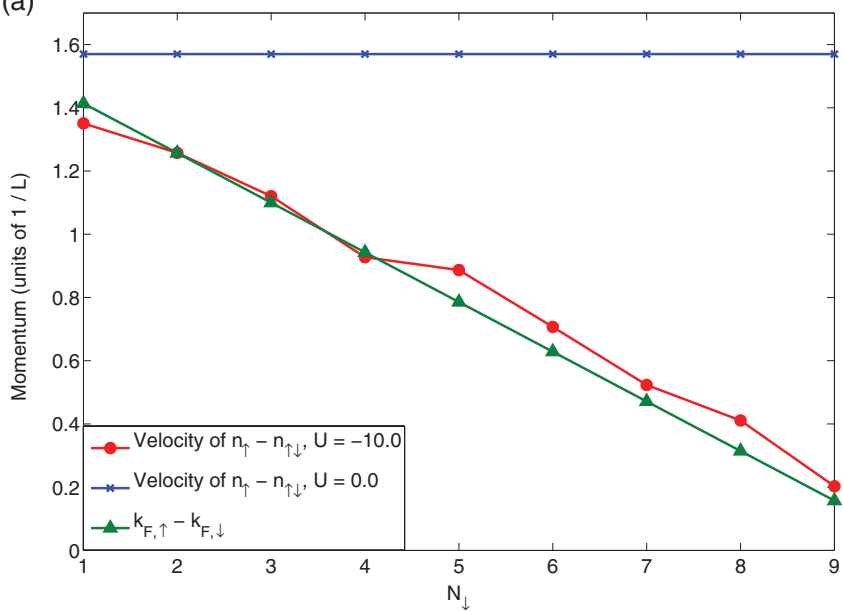

(b)

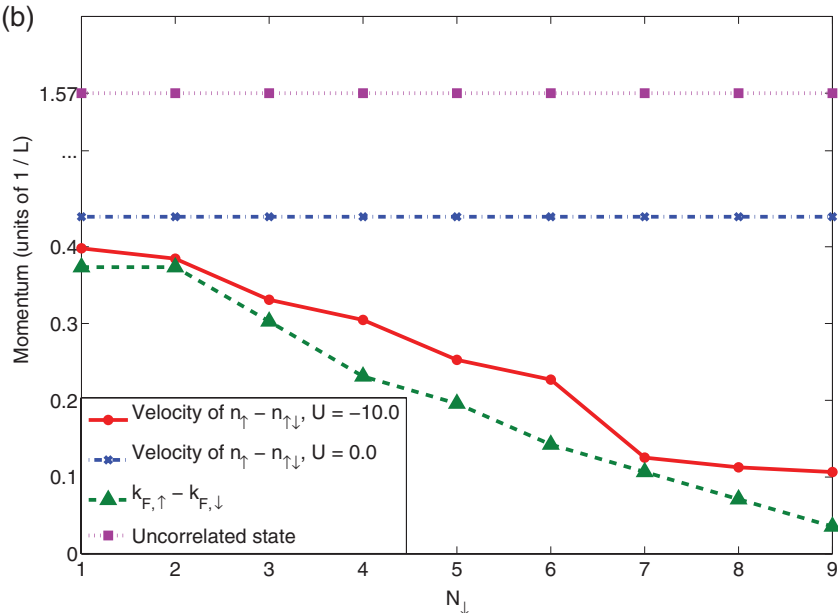

FIG. 4. (Color online) (a) The FFLO momentum $q$ determined from the edge expansion velocity of the unpaired cloud, compared to the $q$ of the ground state, as a function of $N_{\downarrow}$ describing the imbalance $\left(N_{\uparrow}=10\right)$. We also show the expansion momentum $k \neq q$ obtained in the case of a noninteracting gas, and a non-FFLO state without pair coherence. (b) Same as in (a), but having initially a shallow harmonic $\operatorname{trap}\left(V_{h o}=0.0003, C=75.5\right)$.

maximum gradient of the density (see supplementary material, Fig. 1 [25]), one obtains the FFLO momentum from

$$
q=\arcsin \left(\frac{v_{\text {exp }}}{2 J}\right) .
$$

The momentum $q$ obtained in this way from our simulations is compared in Fig. 4(a) to the FFLO momentum as given by the definition $q=k_{F, \uparrow}-k_{F, \downarrow}$ [and by the peak in the momentum pair correlation function of the ground state; cf. Fig. 2(b)]. For reference, we show the expansion velocities in the cases of a noninteracting gas, and a non-FFLO state without pair coherence (discussed later in the text). Only in the case of the FFLO state does the expansion velocity depend on the imbalance. The $q$ extracted from the simulations via Eq. (4) matches excellently the expected FFLO momentum.

In the experimentally relevant case of a harmonic trap, $q$ can be determined in the same way as discussed above. Figure 4(b) shows the comparison of $q$ obtained from the edge expansion velocity to the FFLO momentum in a harmonic trap. The time
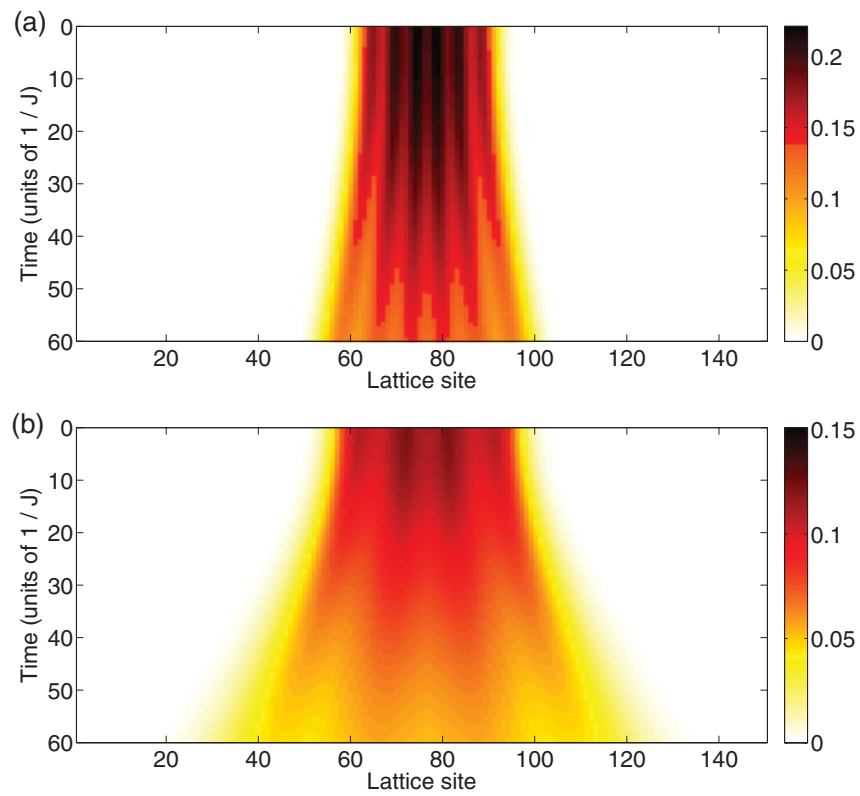

FIG. 5. (Color online) (a) The time development of the doublon density $n_{\uparrow \downarrow}$ with the same parameters as in Fig. 2 but the initial trap being harmonic ( $V_{h o}=0.0003, C=75.5$ ) instead of a box trap. (b) The time development of the unpaired particle density $n_{\uparrow}-n_{\uparrow \downarrow}$.

evolution is shown in Fig. 5. In $q=k_{F \uparrow}-k_{F \downarrow}$, the Fermi momenta are the highest momenta in the harmonic oscillator eigenstates of the quantum numbers $n_{F \uparrow}$ and $n_{F \downarrow}$, respectively (see [25]). In the limit of a shallow trap and large particle number, one approaches the box potential case and a local density approximation argument can be used for the extraction of $k_{F \sigma}$ [26].

The interpretation of our numerical results in terms of the expansion of two fluids is supported by a rigorous Bethe-ansatz analysis of the one-dimensional Fermi-Hubbard Hamiltonian $[22,23,27]$. In the strong-coupling limit, the system can be described as two weakly interacting spinless Fermi gases, corresponding to paired and unpaired particles whose maximum group velocities are the respective Fermi velocities. We thus expect the time evolution of the system to be approximately generated by the free-particle Hamiltonians for paired and unpaired particles. In the case of a lattice model, the group velocity for each momentum component is $v_{k}=2 \tilde{J} \sin [k]$, where the constant $\tilde{J}$ depends on the nature of the particle considered. In our paired and unpaired particle two-fluid model stemming from the Hubbard Hamiltonian, we have $\tilde{J}=J$ for the unpaired particles and $\tilde{J}=2 J^{2} / U$ for the paired particles. For $k<\pi / 2$, the relation $v_{k}=2 \tilde{J} \sin [k]$ thus allows to establish a connection between the maximal expansion velocity and the Fermi momentum of each component (note that for $k \geqslant \pi / 2$ the maximal expansion velocity is given by $2 J$ ).

Based on our numerical findings, we now assume that the unpaired-particle Fermi momentum and the FFLO momentum share the same value $q=k_{F \uparrow}-k_{F \downarrow}$. This is supported by Bethe-ansatz analysis in the continuum case [28] and is also intuitive: in the limit of strong interactions the pair kinetic energies are small and it is energetically favorable that the ground-state structure allows the lowest momentum states up to $q$ to be mainly occupied by unpaired majority particles. Thus 
measuring the maximal expansion velocity of the unpaired particles gives access to the value of the FFLO momentum. As seen in Fig. 4, this scenario agrees with the numerical results, providing a clear signature of the FFLO state. The lattice reproduces the continuum-case dynamics for low densities [16,22] (we have tested also the low-density limit), and our proposal is thus expected to be suitable for the detection of the FFLO state in experiments of the type in [9]. Moreover, the proposed method should be robust with respect to averaging over an array of 1D tubes (cf. [9]).

Our simulations describe the zero-temperature case. Quantum Monte Carlo calculations suggest that there is a finitetemperature precursor for the FFLO state which involves pairing but no FFLO-type correlations [29]. Is the signature we propose for the FFLO state distinguishable from any traces of a state with pairing but no FFLO correlations? Simulating exact dynamics at finite temperature is a formidable task and well beyond the state of the art. However, to consider pairing without FFLO correlations, we have simulated the dynamics of various initial states which have the same average densities of unpaired and paired particles as the corresponding FFLO states, but which possess no correlations between the particles or between the pairs. Such an uncorrelated state can be written as an ensemble of product states, in which each of the product states has completely spatially localized pairs and single majority particles. Choosing the constituents of the ensemble randomly and giving them equal weight, we obtain the results shown in Fig. 4: the expansion velocity does not depend on the polarization. It is simply given by $2 J$ and $4 J^{2} / U$ for unpaired and paired particles, respectively. Therefore, observing a change of the unpairedparticle expansion velocity with the polarization, such that it has the functional dependence $q=k_{F \uparrow}-k_{F \downarrow}$, is a genuine signature of the FFLO correlations and cannot be achieved for a noncorrelated, paired state with the same imbalance. When lowering the temperature, emergence of a $q$-dependent expansion front on top of a thermal-state background would reveal that the FFLO state has been reached.

To conclude, we have shown that, as in many classic ultracold-gas experiments and also in the case of the longsought-for FFLO state, the expansion of the cloud gives an exceedingly simple way of determining the nature of the initial state. Our exact quantum many-body simulations of the 1D imbalanced gas present a clear two-fluid behavior with characteristic expansion velocities. We show that the matching of the expansion velocity of the unpaired majority cloud with the expected FFLO momentum provides an unambiguous signature of the FFLO state.

We thank R.G. Hulet for useful discussions. This work was supported by the Academy of Finland (Projects No. 213362, No. 217043, No. 217045, No. 210953, No. 135000, and No. 141039) and ERC (Grant No. 240362-Heattronics) and was conducted as a part of a EURYI scheme grant. Computing resources were provided by CSC, the Finnish IT Centre for Science.
[1] M. H. Anderson et al., Science 269, 198 (1995).

[2] M. R. Andrews et al., Science 275, 637 (1997).

[3] M. Greiner et al., Nature (London) 415, 39 (2002).

[4] K. O'Hara et al., Science 298, 2179 (2002).

[5] C. Menotti, P. Pedri, and S. Stringari, Phys. Rev. Lett. 89, 250402 (2002).

[6] H. A. Radovan et al., Nature (London) 425, 51 (2003).

[7] A. Bianchi, R. Movshovich, C. Capan, P. G. Pagliuso, and J. L. Sarrao, Phys. Rev. Lett. 91, 187004 (2003).

[8] R. Casalbuoni and G. Nardulli, Rev. Mod. Phys. 76, 263 (2004).

[9] Y.-A. Liao et al., Nature (London) 467, 567 (2010).

[10] P. Fulde and R. A. Ferrell, Phys. Rev. 135, A550 (1964).

[11] A. I. Larkin and Y. N. Ovchinnikov, Zh. Eksp. Teor. Fiz. 47, 1136 (1964) [Sov. Phys. JETP 20, 762 (1965)].

[12] S. Takada and T. Izuyama, Prog. Theor. Phys. 41, 635 (1969).

[13] A. E. Feiguin and F. Heidrich-Meisner, Phys. Rev. B 76, 220508 (2007).

[14] G. G. Batrouni, M. H. Huntley, V. G. Rousseau, and R. T. Scalettar, Phys. Rev. Lett. 100, 116405 (2008).

[15] M. Rizzi et al., Phys. Rev. B 77, 245105 (2008).

[16] K. Yang, Phys. Rev. B 63, 140511 (2001).

[17] G. Orso, Phys. Rev. Lett. 98, 070402 (2007).
[18] M. M. Parish, S. K. Baur, E. J. Mueller, and D. A. Huse, Phys. Rev. Lett. 99, 250403 (2007).

[19] M. W. Zwierlein et al., Science 311, 492 (2006).

[20] G. B. Partridge et al., Science 311, 503 (2006).

[21] M. R. Bakhtiari, M. J. Leskinen, and P. Törmä, Phys. Rev. Lett. 101, 120404 (2008); A. Korolyuk, F. Massel, and P. Törmä, ibid. 104, 236402 (2010); J. M. Edge and N. R. Cooper, Phys. Rev. A 81, 063606 (2010); M. Swanson, Y. L. Loh, and N. Trivedi, e-print arXiv:1106.3908v1.

[22] E. Zhao and W. V. Liu, Phys. Rev. A 78, 063605 (2008).

[23] E. H. Lieb and F. Y. Wu, Phys. Rev. Lett. 20, 1445 (1968); F. H. L. Essler et al., The One-Dimensional Hubbard Model (Cambridge University Press, Cambridge, 2005).

[24] A. J. Daley et al., J. Stat. Mech. Theory Exp. (2004) P04005.

[25] See Supplemental Material at http://link.aps.org/supplemental/ 10.1103/PhysRevA.84.041601 for additional analysis and details.

[26] M. Tezuka and M. Ueda, Phys. Rev. Lett. 100, 110403 (2008).

[27] T. Giamarchi, Quantum Physics in One Dimension (International Series of Monographs on Physics) (Oxford University Press, New York, 2004).

[28] N. Oelkers et al., J. Phys. A Math. Gen. 39, 1073 (2006).

[29] M. Wolak et al., Phys. Rev. A 82, 013614 (2010). 